\title{
Role of preoperative cardiac CT in the evaluation of infective endocarditis: comparison with transesophageal echocardiography and surgical findings
}

\author{
Srikanth Koneru ${ }^{1,2}$, Steven S. Huang ${ }^{3}$, Jorge Oldan ${ }^{1,4}$, Jorge Betancor ${ }^{1}$, Zoran B. Popovic ${ }^{1}$, L. Leonardo \\ Rodriguez $^{1}$, Nabin K. Shrestha ${ }^{5}$, Steven Gordon ${ }^{5}$, Gosta Pettersson ${ }^{6}$, Michael A. Bolen ${ }^{3}$ \\ ${ }^{1}$ Department of Cardiovascular Medicine, Cleveland Clinic, Cleveland, OH, USA; ${ }^{2}$ Division of Cardiology, Department of Medicine, Baylor College \\ of Medicine, Houston, TX, USA; ${ }^{3}$ Department of Diagnostic Radiology, Cleveland Clinic, Cleveland, USA; ${ }^{4}$ Department of Radiology, University \\ of North Carolina, Chapel Hill, USA; ${ }^{5}$ Department of Infectious Disease, ${ }^{6}$ Department of Thoracic and Cardiovascular Surgery, Cleveland Clinic, \\ Cleveland, USA \\ Contributions: (I) Conception and design: S Koneru, J Oldan, MA Bolen; (II) Administrative support: None; (III) Provision of study materials \\ or patients: NK Shrestha; (IV) Collection and assembly of data: S Koneru, J Oldan, J Betancor, SS Huang, MA Bolen; (V) Data analysis and \\ interpretation: S Koneru, J Oldan, ZB Popovic, SS Huang, LL Rodriguez, MA Bolen; (VI) Manuscript writing: All authors; (VII) Final approval of \\ manuscript: All authors. \\ Correspondence to: Michael A. Bolen, MD. Department of Diagnostic Radiology, Cleveland Clinic, 9500 Euclid Ave/J1-4, Cleveland, OH 44195, USA. \\ Email: bolenm@ccf.org.
}

Background: Significant improvement of computed tomography (CT) technology in the last decade has led to more use of this modality for evaluating infective endocarditis (IE) especially since the introduction of high resolution electrocardiogram (ECG) synchronized multiphasic (4D) acquisition. While there are a number of reports on the accuracy and value of 4D CT for evaluation of IE, there is no published data regarding the performance of single-phase ECG gated CT for assessment of IE. The purpose of this study is to evaluate the sensitivity and specificity of preoperative single-phase ECG-gated CT imaging versus transesophageal echocardiography (TEE) in the assessment of complications related to IE, with comparison to surgical findings.

Methods: Among 899 patients with surgically proven IE in our database, 122 underwent contrast-enhanced ECG cardiac CT and were included in the study; 84 of these patients also underwent TEE.

Results: Overall, there was no significant difference between CT and TEE in the identification of pseudoaneurysm/abscess and dehiscence. For the detection of pseudoaneurysm/abscess in prosthetic valves, CT demonstrated higher sensitivity ( $81 \%$ vs. 64\%) and specificity (75\% vs. 33\%) in patients with mechanical aortic valves; TEE demonstrated marginally higher sensitivity (72\% vs. 63\%) and specificity ( $80 \%$ vs. $73 \%)$ in patients with bioprosthetic aortic valves, although the differences are not statistically significant. TEE demonstrated significantly higher sensitivity ( $85 \%$ vs. 16\%) in identifying vegetation in all patients $(\mathrm{P}<0.0001)$, including patients with prosthetic valves (sensitivity, $78 \%$ vs. $19 \%)$. The combined imaging findings of CT and TEE demonstrated improved sensitivity in identifying pseudoaneurysm/abscess and slightly improved detection of prosthesis dehiscence.

Conclusions: Preoperative single-phase gated CT can be seen as complementary to TEE in assessing complications of suspected IE or may be substituted for TEE when vegetation or dehiscence is depicted on transthoracic echocardiography and the patient has a contraindication to TEE.

Keywords: Computed tomography (CT); transesophageal echocardiography (TEE); endocarditis

Submitted Feb 08, 2018. Accepted for publication May 31, 2018.

doi: $10.21037 / \mathrm{cdt} .2018 .07 .07$

View this article at: http://dx.doi.org/10.21037/cdt.2018.07.07 


\section{Introduction}

Infective endocarditis (IE) affects approximately 15,000 individuals in the United States annually. The diagnosis of IE is devastating with up to $40 \%$ risk of mortality (1). Early recognition and accurate diagnosis is paramount for the care of patients with IE who will often need surgery in addition to antibiotic therapy. Transesophageal echocardiography (TEE) is typically the imaging study of choice to assess valvar involvement in IE (2). TEE offers excellent temporal and spatial resolution and is free of ionizing radiation. The important role of TEE in diagnosing IE has been enshrined in the modified Duke criteria (3). In contrast, computed tomography (CT), typically plays a supportive role in imaging assessment of the heart and valves. However, use of this modality for evaluating IE has been increasing especially with the introduction of high resolution electrocardiogram (ECG) synchronized multiphasic (4D) acquisition (4-9). Recently, The European Society of Cardiology incorporated the use of cardiac CT in its guideline for management of IE (10).

At our institution, patients with planned cardiothoracic surgery often undergo an ECG-synchronized CT examination of the thoracic aorta to aid in preoperative planning (routinely in cases of repeat sternotomy). Typically, we acquire single phase ECG-synchronized preoperative CT images for the assessment of the proximity of cardiovascular structures to retrosternal regions and the presence and extent of aortic atheroma. Additionally, complications related to IE may also be evaluated on these CT images, with implications for clinical management. Compared to 4D-CT, these single-phase ECG-gated CT images are easier to acquire, relatively operator independent and require less patient preparation; these exams can be requested and performed at any time in our institution. While there are a number of reports on the accuracy and value of $4 \mathrm{D}-\mathrm{CT}$ for evaluation of IE, there is no published data regarding the performance of single-phase ECG gated CT for assessment of IE (4-6,11). In this investigation, we sought to evaluate the role of CT imaging in the preoperative assessment of patients with established IE and to compare the results of CT with those of TEE and intraoperative findings.

\section{Methods}

Institutional Review Board approval was obtained for this retrospective review with a waiver of informed consent.
We retrospectively searched an institutional database of surgically confirmed IE cases for the presence of contrastenhanced ECG-synchronized CT scans. Inclusion criteria were as follows: (I) patients had surgically proven IE on gross examination and pathologically proven IE with positive causative organism on tissue culture and microscopic examination; and (II) patients had a contrast enhanced ECG gated CT study (either prospective or retrospective synchronization) for pre-surgical evaluation.

Exclusion criteria: (I) patients with negative culture and pathology findings from surgical tissue; (II) patients with non-contrast enhanced and/or non-ECG gated CT scan for pre-surgical evaluation; (III) patients with CT scan performed at an outside institution.

All patients had intraoperative TEE examinations, however these reports were not included in final analysis to avoid bias introduced by surgical feedback at time of acquisition. To evaluate true clinical performance of both CT and TEE imaging, we assessed only initial imaging reports generated before surgery in this investigation, to avoid bias potentially introduced by operating room interactions. Only TEE studies performed and/or interpreted at our hospital were included in the analysis.

\section{Patients}

Of the 899 IE cases that were surgically and pathologically confirmed in the existing database, 677 patients had no preoperative cardiac CT performed, and these patients were excluded from our study. Another 100 patients who had undergone non-contrast-enhanced CT scans and/or non-ECG-gated CT scans were also excluded. This left a total of 122 patients with contrast-enhanced ECG-gated CT scans performed during the patient's hospitalization or within the preceding week as part of preoperative assessment. The time period covers July, 2007 to March, 2014. The patient selection process is summarized in (Figure 1). The clinical characteristics of all study patients are summarized in (Table 1).

A total of 141 valves were evaluated from these 122 patients, including 19 patients with IE involving more than 1 valve (Table 2). Of these patients, 84 had also undergone inhouse preoperative TEE during hospitalization or within the preceding week as part of the evaluation for IE (Figure 1). To evaluate true clinical performance of CT and TEE imaging, the primary analysis was based upon initial imaging reports generated by these studies prior to surgery, so as to avoid potential bias introduced by operating room interaction. 


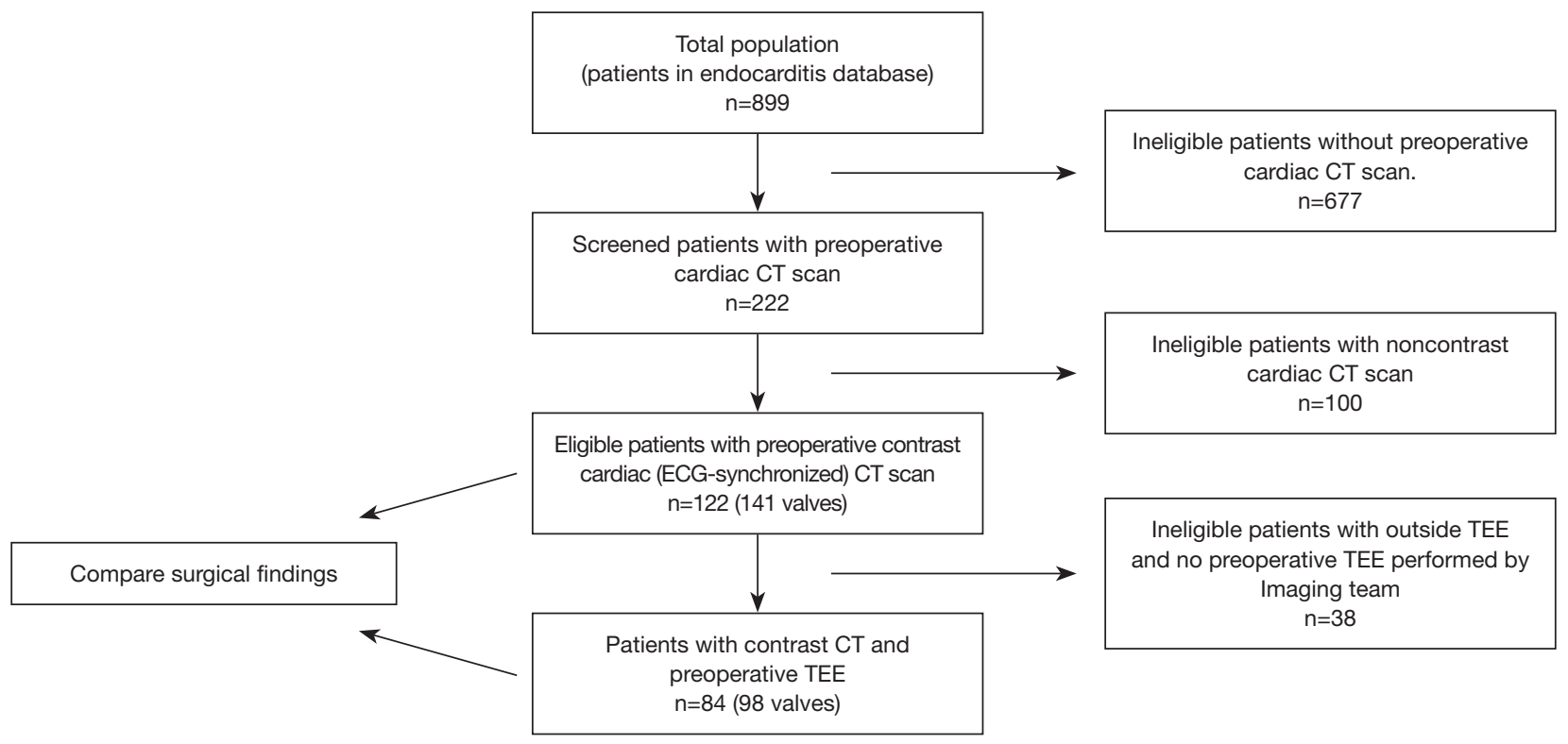

Figure 1 Study protocol.

Table 1 Demographics $(\mathrm{n}=122)$

\begin{tabular}{lc}
\hline Variable & Patients, $\mathrm{n}[\%]$ \\
\hline Male & $83[68]$ \\
History of smoking & $20[16]$ \\
Dyslipidemia & $80[66]$ \\
Dialysis & $8[7]$ \\
Diabetes & $35[29]$ \\
Chronic lung disease & $20[16]$ \\
Chronic kidney disease & $25[20]$ \\
Chronic kidney disease, on dialysis & $9[7]$ \\
Peripheral vascular disease & $9[7]$ \\
Cerebrovascular accident & $25[20]$ \\
Liver disease & $4[3]$ \\
Prior mediastinal radiation & $2[2]$ \\
\hline
\end{tabular}

The median interval between the CT and TEE is 2 days. The median interval between CT and surgery is 4 days. The median interval between TEE and surgery is 6 days.

All study patients had surgical verification of IE involving the mitral and/or aortic valve, with causative organisms reviewed in each case (Table S1). CT findings, including evidence of pseudoaneurysm, abscess, vegetation, and dehiscence were compared with TEE findings when
Table 2 Valve characteristics of patients $(n=122)$

\begin{tabular}{lc}
\hline Valves involved & Patients, $\mathrm{n}$ \\
\hline Native & {$[\%]$} \\
Native aortic and mitral valve & $729]$ \\
Prosthetic & $87[71]$ \\
Mechanical & $26[30]$ \\
Bioprosthetic & $49[56]$ \\
Bioprosthetic aortic valve and native mitral valve & $5[6]$ \\
Bioprosthetic aortic valve and bioprosthetic mitral & $4[5]$ \\
valve & \\
Mechanical aortic valve and mechanical mitral & $1[1]$ \\
valve & \\
Bioprosthetic aortic valve and mechanical mitral & $1[1]$ \\
valve & \\
Bioprosthetic aortic valve and native tricuspid valve & $1[1]$ \\
Prosthetic valve position & $87[71]$ \\
Prosthetic aortic valve & $63[72]$ \\
Prosthetic mitral valve & $12[14]$ \\
Prosthetic aortic valve and prosthetic mitral valve & $6[7]$ \\
Prosthetic aortic valve and native mitral valve & $5[6]$ \\
Prosthetic aortic valve and native tricuspid valve & $1[1]$ \\
\hline Pa & \\
\hline
\end{tabular}

Data are the number of patients, with percentages in parentheses. 

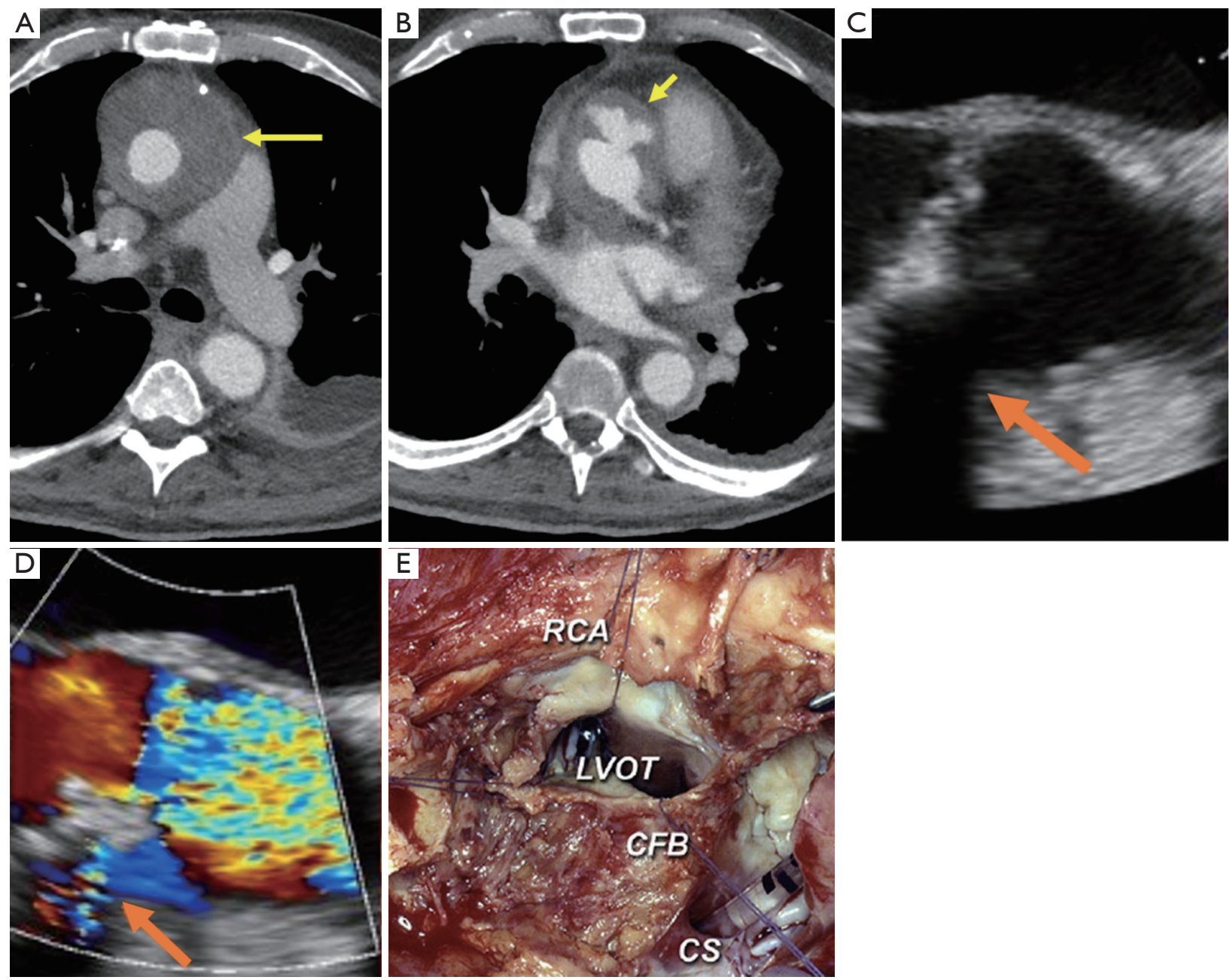

Figure 2 Endocarditis complicated by periaortic abscess [yellow arrow on CT image (A)] and pseudoaneurysm [short yellow arrow on CT (B) and orange arrows on TEE without color Doppler (C) and with color Doppler (D)]. Intraoperative image demonstrates extensive periaortic soft tissue thickening with diffuse inflammation posteriorly (E). Surgical images reprinted with permission from Elsevier (12). RCA, right coronary artery; LVOT, left ventricular outflow tract; CS, coronary sinus; CFB, central fibrous body.

applicable, with surgical reports used as the gold standard. We have combined pseudoaneurysm and abscess as a single positive finding for the purpose of analysis, as the echocardiographic reports did not distinguish between these 2 entities in many instances. Imaging findings were analyzed separately based upon valve type (prosthetic and native) and anatomic position.

We defined the following imaging criteria for IE complications.

\section{CT imaging}

\section{Pseudoaneurysm}

Contrast filled smooth walled sac adjacent to vascular structure or heart, usually with a visible direct connection (Figure 2).

\section{Abscess}

Low attenuation central necrotic component within a peripheral organized rim, which may be irregular and thick, with enhancement by contrast. Surrounding inflammation and mass effect may also be present. Diffuse soft tissue thickening surrounding cardiac or aortic structures (which may represent phlegmon/early abscess) were also placed in this category (Figure 2).

\section{Dehiscence}

Malalignment of prosthesis with tissue defect between annulus and prosthesis (Figure 3). 

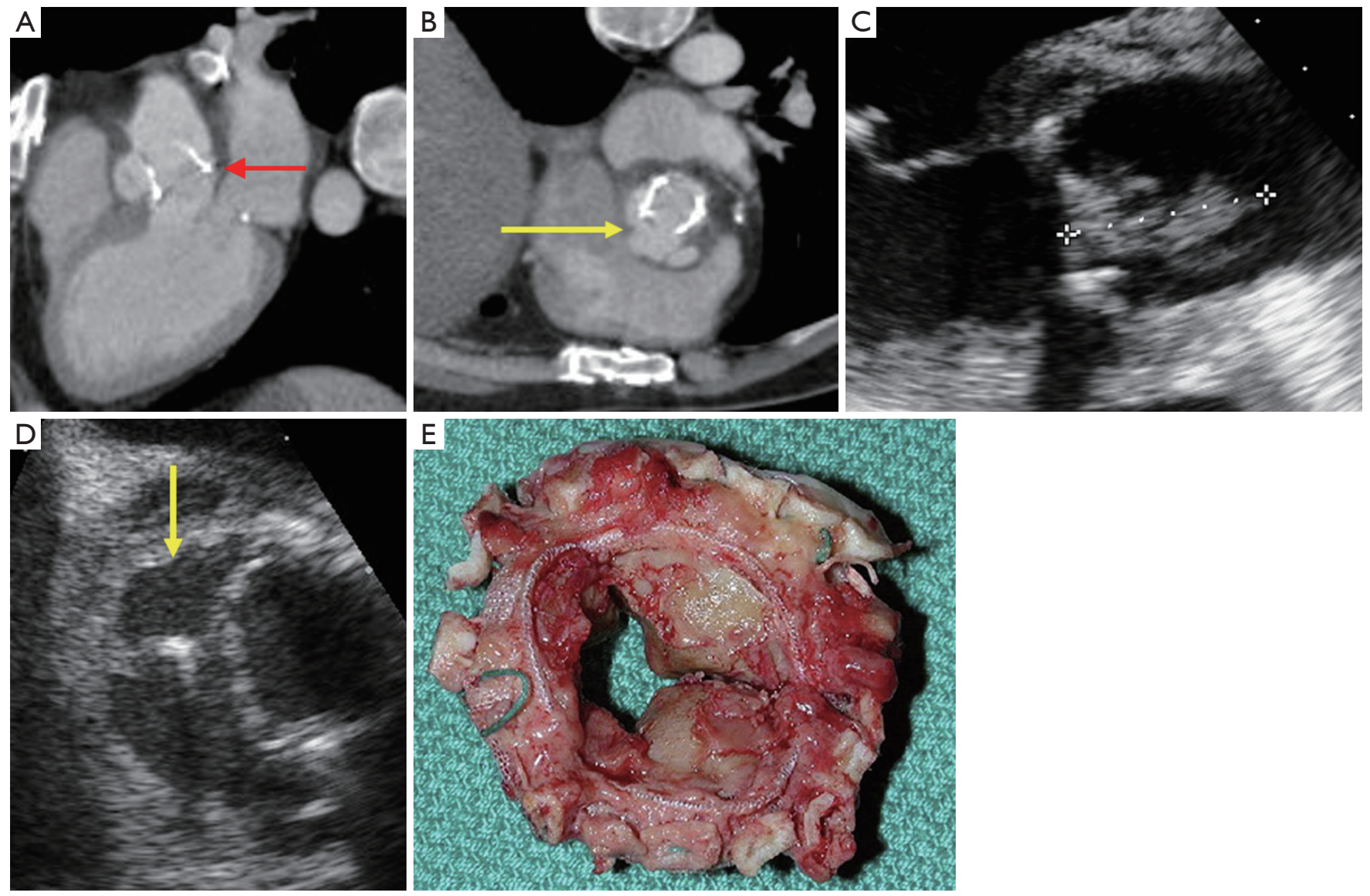

Figure 3 Dehiscence of a bioprosthetic aortic valve in setting of endocarditis complicated by pseudoaneurysm (red and yellow arrows from three chamber (A) and short axis (B) CTA reformats, yellow arrow from TEE (D). Vegetation noted as well (C). Explanted partially dehisced bioprosthesis (E) infected with Staphylococcus aureus. Surgical images reprinted with permission from Elsevier (12). TEE, transesophageal echocardiography.

\section{Vegetation}

Low to intermediate attenuation irregular mass or prominent focal thickening associated with valve, endocardium, or prosthesis (Figure 4).

\section{TEE}

\section{Pseudoaneurysm/abscess}

Region of reduced echodensity or discrete echolucent cavity in the annular region or adjacent myocardium or vascular structures. In the setting of pseudoaneurysm to-and-fro color flow may be demonstrated within its cavity, and a visible direct connection may be demonstrated (Figure 2).

\section{Dehiscence}

Rocking motion of prosthesis, possibly with tissue defect between annulus and prosthesis or paravalvular flow on Doppler assessment (Figure 3).

\section{Vegetation}

Independently mobile echodense mass associated with valve, endocardium, or prosthesis (Figure 4).

\section{CT image acquisition}

CT scans were obtained on a Phillips iCT $2 \times 128$ slice scanner (Phillips Medical Devices, Best, the Netherlands), Phillips Brilliance 64-slice scanner, Siemens Somatom Definition Flash $2 \times 128$ slice scanner (Siemens Healthcare, Erlangen, Germany), Siemens Definition $2 \times 64$ scanner, Siemens Sensation 64 scanner and Siemens Sensation 16 scanner. Numbers of patients scanned on each instrument are presented in Table S2. ECG synchronization was retrospective for all inpatients (based on scanner limitations); outpatients were typically imaged with 

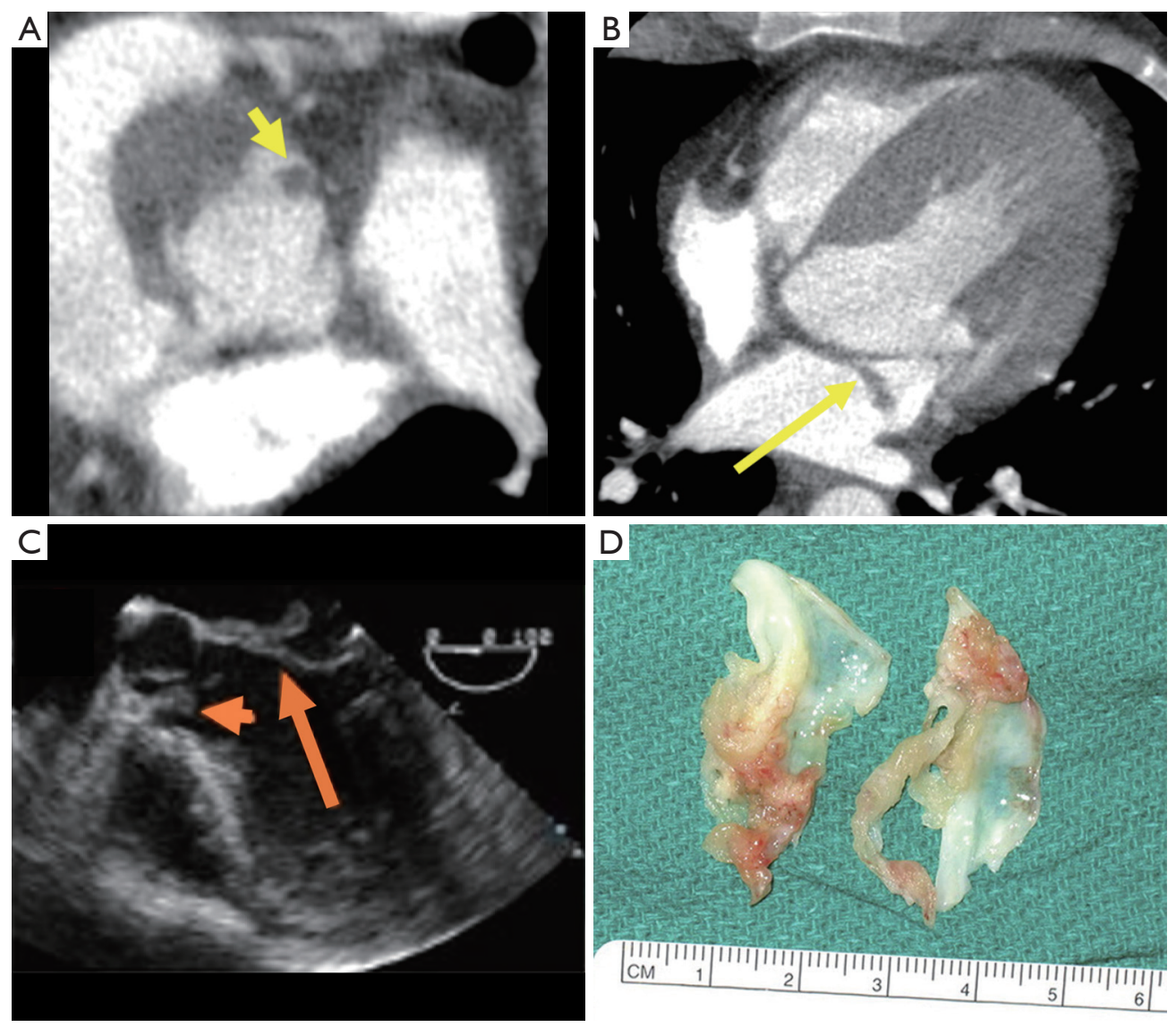

Figure 4 Vegetations associated with endocarditis noted on native aortic and mitral valve leaflets [short and long yellow arrows on CT images (A,B), short and long orange arrows on TEE image (C)]. Excised aortic valve cusps (D) with vegetations and cusp degeneration. Surgical images reprinted with permission from Elsevier (12). TEE, transesophageal echocardiography.

prospective triggering. Scanner $\mathrm{kV}$ and $\mathrm{mAs}$ settings were determined by patient body habitus and internally developed software. A weight-based dosing regimen of intravenous contrast (Ultravist 370; 3 patients at the beginning of the period received Isovue, and 9 at the end received Omnipaque) was used. Images were reconstructed to a slice thickness of $3 \mathrm{~mm}$ and an increment of $3 \mathrm{~mm}$. Images were analyzed at dedicated workstations by physicians with cardiovascular imaging fellowship training. Either Agfa IMPAX 6 (Mortsel, Belgium) or TeraRecon Aquarius iNtuition (Foster City, CA, USA) software was used for image analysis. Clinical imaging reports directed toward identification of relevant preoperative findings (aortic size, presence and extent of atheroma, proximity of cardiac and vascular structures to midline retro-sternum) as well as any incidental abnormality was reviewed.

\section{Statistical analysis}

Statistical analyses were performed using MATLAB 2009. Sensitivity, specificity, negative predictive values (NPVs), and positive predictive values (PPVs) for pseudoaneurysm/ abscess, dehiscence, and vegetation were calculated using standard methodology for cases with definitive answers. McNemar's test was used to evaluate for significant differences between modalities. A P value $<0.05$ was indicative of statistical significance for all analyses.

To assess the accuracy of combined (TEE + CT) imaging findings, we analyzed the sensitivity and specificity of each finding. Findings were defined as true positive if either or both of the imaging modalities was positive compared to positive surgical findings, true negative if both of the imaging modalities were negative compared to negative surgical findings, false positive if either or both of the 
Table 3 Evaluation of pseudoaneurysm/abscess, vegetation, and dehiscence by modality

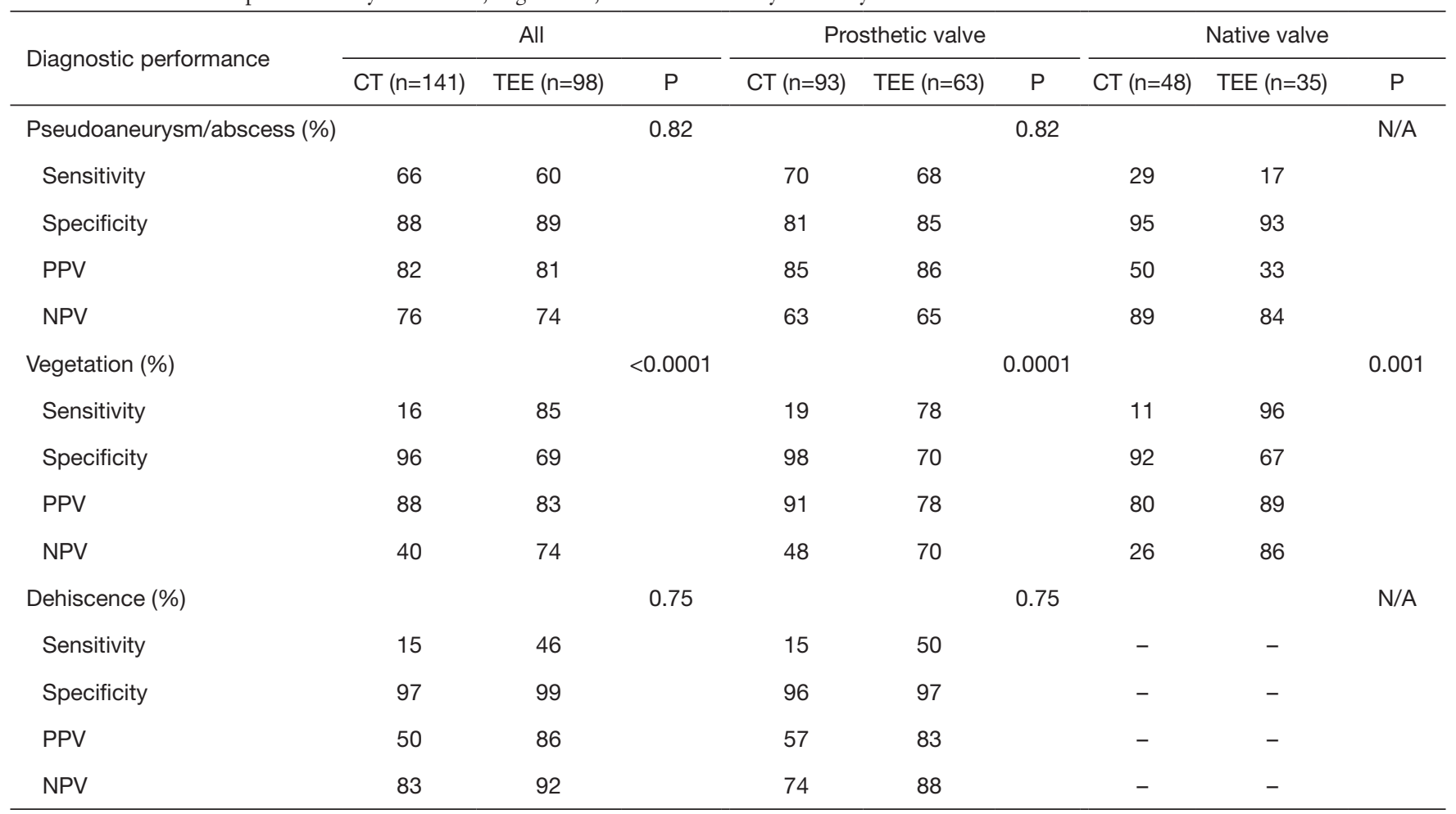

CT, computed tomography; N/A, not applicable; NPV, negative predictive value; PPV, positive predictive value; TEE, transesophageal echocardiograph.

imaging modalities were positive compared to negative surgical findings, or false negative if both of the modalities were negative compared to positive surgical findings.

Additionally, Kappa Fleiss inter-reader agreement analysis was performed in 25 randomly selected cases, which were retrospectively re-analyzed by three separate readers to assess both CT and TEE findings independently. Findings made by the three readers were compared for the inter-reader agreement analysis.

\section{Results}

All patients had surgically verified findings suggestive of endocarditis, with a total of 76 patients with valvular vegetation, 63 patients with either abscess or pseudoaneurysm, and 23 patients with valve dehiscence. These findings represented the gold standard and were compared to findings reported on TEE and CT imaging.

\section{TEE versus CT for detection of pseudoaneurysm/abscess}

The diagnostic accuracy of CT for the detection of pseudoaneurysm/abscess was similar to the accuracy of TEE when all cases were analyzed $(\mathrm{P}=0.82$; Table 3).

In subgroup analysis of patients with native valve IE, the diagnostic accuracy of CT in detecting pseudoaneurysm/ abscess was also similar to the accuracy of TEE.

In patients with prosthetic valve IE, there was no significant difference between CT and TEE in the detection of pseudoaneurysm/abscess $(\mathrm{P}=0.82$; Table 3). Overall, there was no statistical difference between CT and TEE in detecting pseudoaneurysm/abscess in patients with bioprosthetic or mechanical valves when valve location was considered $(\mathrm{P}=1$ for aortic valve and $\mathrm{P}=0.47$ for mitral valve) (Tables $S 3$ and $S 4$ ). We noted slightly better sensitivity ( $81 \%$ vs. $64 \%$ ) and specificity (75\% vs. $33 \%$ ) with CT than with TEE in detecting pseudoaneurysm/abscess in patients with mechanical aortic valve IE, whereas TEE showed marginally better sensitivity (72\% vs. $63 \%)$ and specificity $(80 \%$ vs. $73 \%)$ than CT in detecting pseudoaneurysm/abscess in patients with bioprosthetic aortic valves (Table S3). However, the differences did not reach statistical significance in this study $(\mathrm{P}=0.68$ and $\mathrm{P}=1$ respectively). 


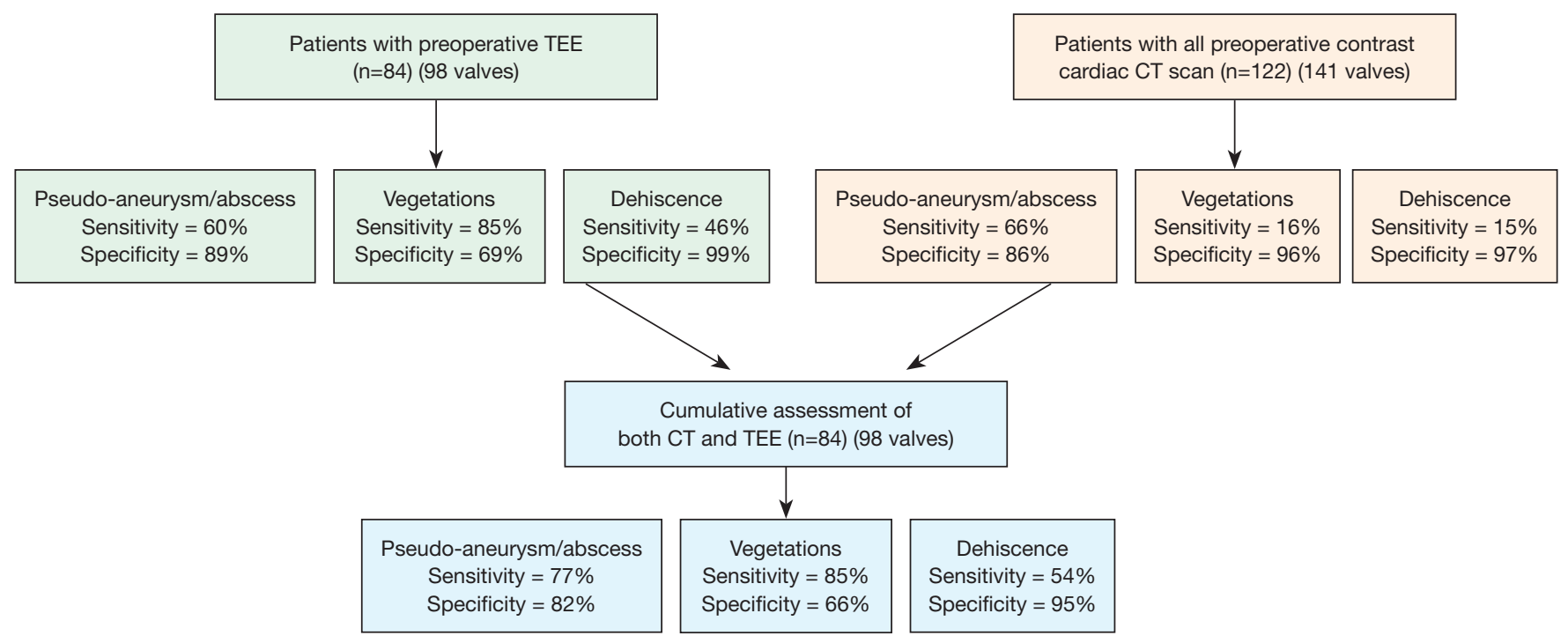

Figure 5 The sensitivity and specificity of detecting pseudoaneurysm/abscess, vegetation, and dehiscence in the overall patient population with CT, TEE, and CT + TEE combined. TEE, transesophageal echocardiography.

\section{TEE versus CT for detection of vegetation}

A statistically significant difference in accuracy was noted between CT and TEE for the detection of vegetation $(\mathrm{P}<0.0001)$ among all study patients. TEE had significantly higher sensitivity than CT (85\% vs. $16 \%)$ in detecting vegetation; however, CT showed better specificity than TEE (96\% vs. 69\%; Table 3).

Subgroup analysis of patients with native and prosthetic valves and those with valves in aortic and mitral valve positions demonstrated similar findings, with TEE showing higher sensitivity and CT showing higher specificity in the detection of vegetation (Tables $S 3$ and $S 4$ ).

\section{TEE versus CT for detection of valve dehiscence}

In all study patients, CT and TEE demonstrated similar specificity and NPV (specificity, 97\% vs. 99\%; NPV, $83 \%$ vs. $92 \%$ ) for the detection of valve dehiscence. TEE demonstrated a higher sensitivity and PPV versus CT for the detection of dehiscence (sensitivity, $46 \%$ vs. 15\%; PPV, $86 \%$ vs. 50\%). Overall, there was no significant difference in accuracy between modalities in all study patients $(\mathrm{P}=0.75$; Table 3) or in subgroup analysis (Tables $S 3$ and S4).

\section{Combined analysis of CT + TEE versus surgical findings}

The combined CT + TEE findings demonstrated overall improvement in sensitivity for the detection of pseudoaneurysm/abscess (CT, 66\%; TEE, 60\%; CT + TEE, $77 \%$ ) and dehiscence (CT, 15\%; TEE, 46\%; CT + TEE, 54\%). However, there was no significant change in sensitivity for the detection of vegetation (Figure 5).

In patients with prosthetic valve IE, analysis again demonstrated improved sensitivity with combined CT + TEE in detecting pseudoaneurysm/abscess (CT, 70\%; TEE, 68\%; CT + TEE, 86\%) and dehiscence (CT, 15\%; TEE, 46\%; CT + TEE, 54\%) with no significant change in the detection of vegetation (Figure S1). In a sub-analysis of prosthetic valves in the aortic position, CT + TEE also demonstrated improved sensitivity for the detection of pseudoaneurysm/abscess (CT, 70\%; TEE, 68\%; CT + TEE, $86 \%$ ) and dehiscence (CT, 21\%; TEE, 50\%; CT + TEE, 60\%) (Figure S2).

\section{Inter reader agreement analysis}

Identification of vegetation showed slight to fair interreader agreement on $\mathrm{CT}(\kappa=0.08$ aortic valve, $\kappa=0.37 \mathrm{mitral}$ valve) and fair to moderate inter-reader agreement on TEE ( $\kappa=0.31$ aortic valve, $\kappa=0.54$ mitral valve). The assessment of abscess and pseudoaneurysm showed moderate agreement on both CT and TEE (CT $\kappa=0.52$ aortic valve, $\kappa=0.57$ mitral valve; TEE $\kappa=0.68$ aortic valve, $\kappa=0.58$ mitral valve). The evaluation of dehiscence showed no significant 
agreement on CT ( $\kappa=0$ aortic valve, $\kappa=0$ mitral valve), and fair to moderate agreement on TEE ( $\kappa=0.21$ aortic valve, $\kappa=0.64$ mitral valve).

\section{Incidental findings}

TEE identified many incidental common valvular disorders such as regurgitation and stenosis, as well as less common valvular disorders such as perforation, flail leaflet, and prolapse. CT identified a number of extracardiac incidental findings (Table S5). Many of the extracardiac findings identified on CT such as pleural effusions and pneumonia are detectable on a chest radiograph, whereas others such as splenic infarcts may be of questionable clinical importance when IE is already suspected. CT did identify 4 incidental findings that could be considered critical, including a prosthesis dehiscence, contained aortic rupture, pulmonary embolus, and a spiculated pulmonary nodule.

\section{Discussion}

In this study, we compared the real-world clinical imaging performance of preoperative single-phase ECG-gated CT with the performance of TEE in the collection of surgically proven IE cases. We observed no significant difference between CT and TEE in accurately identifying pseudoaneurysm/abscess or dehiscence in overall patient groups. However, TEE demonstrated higher sensitivity in identifying vegetation versus CT in all patients, including patients with prosthetic valves (both bioprosthetic and mechanical), whereas CT demonstrated higher specificity. This is likely due to the near real time nature of echocardiography that aids in the assessment of mobile small lesions and the fact that only static CT images were used in this study. In the assessment of pseudoaneurysm/ abscess, CT demonstrated higher sensitivity and specificity in patients with mechanical aortic valves, whereas TEE had higher sensitivity and specificity in patients with bioprosthetic aortic valves. While the differences did not reach statistical significance, these might be attributable to the less obtrusive pattern of CT artifacts compared to sonographic artifacts associated with mechanical valves. Similar differences are not observed with prosthetic valves in the mitral position; this could be due to the small number of patients with prosthetic mitral valve in this study.

The combined imaging findings of CT and TEE improved the detection of pseudoaneurysm/abscess and dehiscence, possibly due to the combined benefit of
CT volumetric acquisition and decreased artifact, with the improved temporal resolution of echocardiography. Moderate agreement was noted for both TEE and CT in the assessment of abscess/pseudoaneurysm. Interestingly, the assessment of both vegetation and dehiscence by TEE showed only fair to moderate agreement, possibly limited by hardware related artifact.

Literature values for the sensitivity of TEE in detecting vegetations have ranged from $87 \%$ to $100 \%$ (5-9), although some smaller studies have reported lower values (13). TEE is generally considered sensitive for the detection of abscess; although one study of 115 patients reported a sensitivity of $48 \%$ (14), most studies have demonstrated sensitivity values ranging from $80 \%$ to $90 \%(15-17)$. Fewer studies assessing TEE for the detection of pseudoaneurysm have been performed. A retrospective review of the literature reported a sensitivity of $100 \%$ for TEE in the detection of pseudoaneurysms; however, this result may have been influenced by selection bias, with nonidentified pseudoaneurysms going unreported (18). Large studies evaluating the accuracy of TEE in identifying valvular dehiscence have not been performed, though one group reported a sensitivity value of $100 \%$ for TEE in the detection of dehiscence of aortic grafts (19).

Several studies using 4D-CT have demonstrated accuracy comparable to TEE in diagnosing valvular involvement in IE. Fagman et al. described a retrospective study of 27 patients with prosthetic aortic valve IE in which ECG-gated 4D-CT demonstrated strong agreement with surgical findings, although not as strong as the agreement between TEE and surgical findings (6). Feuchtner $e t$ al. compared ECG-gated 4D-CT with TEE as a gold standard on a mix of valves in 37 patients with IE and observed $97 \%$ sensitivity, $88 \%$ specificity, $97 \% \mathrm{PPV}$, and $88 \% \mathrm{NPV}$ for the detection of valvar abnormalities on a per-patient basis. CT was able to identify most vegetations, abscesses, and pseudoaneurysms, with good correlation between CT and TEE for size measurements and mobility. However, CT was not able to detect leaflet perforations (4). Gahide $e t$ al. studied aortic valves in 19 patients with IE, comparing ECG-gated 4D-CT with intraoperative findings. In this study, CT demonstrated $100 \%$ sensitivity, $87.5 \%$ specificity, $91.7 \% \mathrm{PPV}$, and $100 \% \mathrm{NPV}$ for the detection of aneurysms and perfect agreement with surgical findings for the extension of aortic valve pseudoaneurysms into the intervalvular fibrous body (5). However, CT had a sensitivity of only $71.4 \%$ and an NPV of $55.5 \%$ (specificity and PPV were perfect) for the detection of valve vegetations (although 
agreement was perfect for vegetations $>1 \mathrm{~cm})$. Koo et al. recently published a retrospective review of 49 patients who underwent 4-D CT and TEE and demonstrated that 4DCT has $91 \%$ sensitivity for vegetation and $60 \%$ sensitivity for abscess/pseudoaneurysm (9). Another recently published is an article by Sims et al. who conducted a retrospective review of 137 patients that had cardiac CT prior to IE related surgery; majority of these patients had multiphasic 4-D CT (8). Sims et al. demonstrated $90.5 \%$ sensitivity for abscess/pseudoaneurysm and 70\% sensitivity for vegetation.

Comparing to these trials, our single-phase preoperative CT was not as sensitive for the assessment of vegetations. One suspected reason for lower sensitivity is the slice thickness of our images; we reconstruct images with $3 \mathrm{~mm}$ slice thickness. Studies by Gahide et al. and Fagman et al. reported $0.6 \mathrm{~mm}$ slice thickness $(5,6)$. Feuchtner et al. reconstructed $0.7-\mathrm{mm}$ images for end-systole and enddiastole, and reconstructed $1.0 \mathrm{~mm}$ images for the 10 phases-dynamic data set (4). Another contributing factor is likely the single-phase imaging, which limits assessment of mobile entities.

There are a few advantages of our preoperative CT protocol. Optimized 4D cine imaging of the cardiovascular structures typically involves higher radiation doses due to retrospective technique and thin slice reconstruction. Although radiation dose is less of a concern for elderly or critically ill patients, the single-phase protocol with thicker slice reconstruction facilitates lower radiation exposure compared to high resolution 4D-CT protocols utilized by previous investigators. For example, Fagman et al. reported a mean effective dose of $19 \mathrm{mSv}$, whereas we observed a mean estimated dose of $10 \mathrm{mSv}$ using a conversion factor of $0.017 \mathrm{mSv} /(\mathrm{mGy} \mathrm{cm})$ (6). Additionally, patients with rapid heart rates may require use of chronotropic medications to facilitate 4D cine imaging, a possible point of concern in acutely ill or frail patients. Furthermore, our acquisition included the entire thoracic aorta, whereas other investigators such as Feuchtner et al. imaged from the carina to the base of the heart. Our acquisition allowed for preoperative assessment of atheromatous changes throughout the aorta as well as IE complications remote from the valve (4). In our study, CT helped us to identify 4 critical incidental findings. Overall, while there are some relative benefits to our simplified preoperative protocol, we acknowledge some of the limitations associated with single phase imaging and thicker slice reconstruction. This CT protocol was selected as a general preoperative imaging tool for patients with a variety of cardiovascular pathologies. In future investigations we plan to evaluate the benefits of hybridized preoperative CT imaging protocols for patients with suspected endocarditis, with the hopes of capturing benefits of both 4D CT imaging as well as broader anatomic coverage of cardiovascular structures.

Our study had some limitations. TEE was not available for comparison in all cases. The CT scans were performed on many different machines; the different technical aspects of each machine may have introduced systematic errors that are not explored in this study. Similarly, there may be systematic differences between CT scan of inpatients (mostly acquired with retrospective gating) and CT scan of outpatients (mostly acquired with prospective gating). Since this is a retrospective study, the time intervals between CT, TEE and surgery varies among patients. Since IE is a progressive disease, comparison of imaging modalities performed at different time points may not be optimal. Lastly, the results of this investigation are drawn from a cohort referred for surgery and therefore may not be generalizable to all IE cases.

In conclusion, contrast-enhanced ECG-synchronized CT of the thoracic aorta performed for preoperative planning in a large group of patients with suspected IE provided overall similar performance to TEE in the identification of pseudoaneurysm or abscess, with CT possibly providing better assessment in patients with mechanical aortic prostheses and TEE performing better in patients with bioprosthetic aortic valves, although the differences are not statistically significant. TEE was more effective in identifying vegetations among all study patients. TEE demonstrated higher sensitivity and CT demonstrated higher specificity in identifying dehiscence. These findings did not change based on valve location. The combined imaging findings of CT and TEE improved the detection of pseudoaneurysm/abscess and dehiscence. These results suggest that simple single-phase ECG gated CT performed for preoperative planning can be complementary to TEE in assessing patients with suspected IE or could be used as an alternative to TEE when vegetation or dehiscence is seen on transthoracic echocardiography and TEE is contraindicated.

\section{Acknowledgements}

The authors would like to acknowledge Megan Griffiths for her assistance in manuscript preparation. 


\section{Footnote}

Conflicts of Interest: The authors have no conflicts of interest to declare.

Ethical Statement: The study was approved by institutional review board (IRB) of Cleveland Clinic (No. 15-143). A waiver of Informed Consent and waiver of HIPAA authorization are approved to allow access to patient's medical record by the research team.

\section{References}

1. Bashore TM, Cabell C, Fowler V. Update on infective endocarditis. Curr Probl Cardiol 2006;31:274-352.

2. Thuny F, Grisoli D, Cautela J, et al. Infective endocarditis: prevention, diagnosis, and management. Can J Cardiol 2014;30:1046-57.

3. Li JS, Sexton DJ, Mick N, et al. Proposed modifications to the Duke criteria for the diagnosis of infective endocarditis. Clin Infect Dis 2000;30:633-8.

4. Feuchtner GM, Stolzmann P, Dichtl W, et al. Multislice computed tomography in infective endocarditis: comparison with transesophageal echocardiography and intraoperative findings. J Am Coll Cardiol 2009;53:436-44.

5. Gahide G, Bommart S, Demaria R, et al. Preoperative evaluation in aortic endocarditis: findings on cardiac CT. AJR Am J Roentgenol 2010;194:574-8.

6. Fagman E, Perrotta S, Bech-Hanssen O, et al. ECGgated computed tomography: a new role for patients with suspected aortic prosthetic valve endocarditis. Eur Radiol 2012;22:2407-14.

7. Habets J, Tanis W, van Herwerden LA, et al. Cardiac computed tomography angiography results in diagnostic and therapeutic change in prosthetic heart valve endocarditis. Int J Cardiovasc Imaging 2014;30:377-87.

8. Sims JR, Anavekar NS, Chandrasekaran K, et al. Utility of cardiac computed tomography scanning in the diagnosis and pre-operative evaluation of patients with infective endocarditis. Int J Cardiovasc Imaging 2018. [Epub ahead of print].

9. Koo HJ, Yang DH, Kang JW, et al. Demonstration of infective endocarditis by cardiac CT and transoesophageal echocardiography: comparison with intra-operative findings. Eur Heart J Cardiovasc Imaging 2018;19:199-207.

10. Habib G, Lancellotti P, Antunes MJ, et al. 2015 ESC Guidelines for the management of infective endocarditis: The Task Force for the Management of Infective
Endocarditis of the European Society of Cardiology (ESC). Endorsed by: European Association for Cardio-Thoracic Surgery (EACTS), the European Association of Nuclear Medicine (EANM). Eur Heart J 2015;36:3075-128.

11. Fagman E, Flinck A, Snygg-Martin U, et al. Surgical decision-making in aortic prosthetic valve endocarditis: the influence of electrocardiogram-gated computed tomography. Eur J Cardiothorac Surg 2016;50:1165-71.

12. Pettersson GB, Hussain ST, Shrestha NK, et al. Infective endocarditis: an atlas of disease progression for describing, staging, coding, and understanding the pathology. J Thorac Cardiovasc Surg 2014;147:1142-9.e2.

13. Taams MA, Gussenhoven EJ, Bos E, et al. Enhanced morphological diagnosis in infective endocarditis by transoesophageal echocardiography. Br Heart J 1990;63:109-13.

14. Hill EE, Herijgers P, Claus P, et al. Abscess in infective endocarditis: the value of transesophageal echocardiography and outcome: a 5-year study. Am Heart J 2007;154:923-8.

15. Graupner C, Vilacosta I, SanRomán J, et al. Periannular extension of infective endocarditis. J Am Coll Cardiol 2002;39:1204-11.

16. Karalis DG, Bansal RC, Hauck AJ, et al. Transesophageal echocardiographic recognition of subaortic complications in aortic valve endocarditis. Clinical and surgical implications. Circulation 1992;86:353-62.

17. San Román JA, Vilacosta I, Sarriá C, et al. Clinical course, microbiologic profile, and diagnosis of periannular complications in prosthetic valve endocarditis. Am J Cardiol 1999;83:1075-9.

18. Sudhakar S, Sewani A, Agrawal M, et al. Pseudoaneurysm of the mitral-aortic intervalvular fibrosa (MAIVF): A comprehensive review. J Am Soc Echocardiogr 2010;23:1009-18; quiz 1112.

19. Ballal RS, Gatewood RP, Nanda NC, et al. Usefulness of transesophageal echocardiography in the assessment of aortic graft dehiscence. Am J Cardiol 1997;80:372-6.

Cite this article as: Koneru S, Huang SS, Oldan J, Betancor J, Popovic ZB, Rodriguez LL, Shrestha NK, Gordon S, Pettersson G, Bolen MA. Role of preoperative cardiac CT in the evaluation of infective endocarditis: comparison with transesophageal echocardiography and surgical findings. Cardiovasc Diagn Ther 2018;8(4):439-449. doi: 10.21037/ cdt.2018.07.07 


\section{Multivalve involvement}

A total of 19 patients had involvement of $>1$ valve; 7 patients had native valve IE involving mitral and aortic valves, and 12 patients had $\geq 1$ underlying prosthetic valve IE. Of the 5 patients with prosthetic valves in the mitral and aortic positions, 3 patients had both bioprosthetic valves, 1 patient had both mechanical valves, and 1 patient had a mechanical valve in the mitral valve position and a bioprosthetic valve in the aortic valve position. Only 1 patient had both bioprosthetic aortic and tricuspid valves. Of the remaining
6 patients with involvement of at least 1 prosthetic valve, 5 patients had bioprosthetic aortic valves, including 4 with native mitral valves and 1 with a native tricuspid valve. One patient had a mechanical aortic valve and a repaired mitral valve.

\section{Raw data}

The following pages are the $2 \times 2$ tables with actual valve/ patient counts for all of the evaluated categories.

\begin{tabular}{|c|c|c|c|c|c|c|c|c|c|}
\hline All & CT-pseudo or abscess & & & CT-vegetation & & & CT-dehisc & & \\
\hline & True positive & False positive & & True positive & False positive & & True positive & False positive & \\
\hline & 42 & 9 & & 14 & 2 & & 4 & 4 & \\
\hline & 22 & 68 & & 75 & 50 & & 22 & 111 & \\
\hline & False negative & True negative & 141 & False negative & True negative & 141 & False negative & True negative & 141 \\
\hline & TEE-pseudo or abscess & & & TEE-vegetation & & & TEE-dehisc & & \\
\hline & True positive & False positive & & True positive & False positive & & True positive & False positive & \\
\hline & 26 & 6 & & 53 & 11 & & 6 & 1 & \\
\hline & 17 & 49 & & 9 & 25 & & 7 & 84 & \\
\hline & False negative & True negative & 98 & False negative & True negative & 98 & False negative & True negative & 98 \\
\hline
\end{tabular}

\begin{tabular}{|c|c|c|c|c|c|c|c|c|c|}
\hline \multirow[t]{10}{*}{ Native } & CT-pseudo or abscess & & & CT-vegetation & & & CT-dehisc & & \\
\hline & True positive & False positive & & True positive & False positive & & True positive & False positive & \\
\hline & 2 & 2 & & 4 & 1 & & 0 & 1 & \\
\hline & 5 & 39 & & 32 & 11 & & 2 & 32 & \\
\hline & False negative & True negative & 48 & False negative & True negative & 48 & False negative & True negative & 35 \\
\hline & TEE-pseudo or abscess & & & TEE-vegetation & & & TEE-dehisc & & \\
\hline & True positive & False positive & & True positive & False positive & & True positive & False positive & \\
\hline & 1 & 2 & & 25 & 3 & & 0 & 0 & \\
\hline & 5 & 27 & & 1 & 6 & & 0 & 25 & \\
\hline & False negative & True negative & 35 & False negative & True negative & 35 & False negative & True negative & 25 \\
\hline
\end{tabular}

\begin{tabular}{|c|c|c|c|c|c|c|c|c|c|}
\hline \multirow[t]{10}{*}{ Prosthetic } & CT-pseudo or abscess & & & CT-vegetation & & & CT-dehisc & & \\
\hline & True positive & False positive & & True positive & False positive & & True positive & False positive & \\
\hline & 40 & 7 & & 10 & 1 & & 4 & 3 & \\
\hline & 17 & 29 & & 43 & 39 & & 22 & 64 & \\
\hline & False negative & True negative & 93 & False negative & True negative & 93 & False negative & True negative & 93 \\
\hline & TEE-pseudo or abscess & & & TEE-vegetation & & & TEE-dehisc & & \\
\hline & True positive & False positive & & True positive & False positive & & True positive & False positive & \\
\hline & 25 & 4 & & 28 & 8 & & 6 & 1 & \\
\hline & 12 & 22 & & 8 & 19 & & 7 & 49 & \\
\hline & False negative & True negative & 63 & False negative & True negative & 63 & False negative & True negative & 63 \\
\hline
\end{tabular}




\begin{tabular}{|c|c|c|c|c|c|c|c|c|c|}
\hline \multirow[t]{10}{*}{ Total prosthetic-AV } & CT-pseudo or abscess & & & CT-vegetation & & & CT-dehisc & & \\
\hline & True positive & False positive & & True positive & False positive & & True positive & False positive & \\
\hline & 39 & 5 & & 8 & 1 & & 4 & 3 & \\
\hline & 17 & 14 & & 34 & 32 & & 15 & 53 & \\
\hline & False negative & True negative & 75 & False negative & True negative & 75 & False negative & True negative & 75 \\
\hline & TEE-pseudo or abscess & & & TEE-vegetation & & & TEE-dehisc & & \\
\hline & True positive & False positive & & True positive & False positive & & True positive & False positive & \\
\hline & 25 & 4 & & 18 & 6 & & 5 & 1 & \\
\hline & 12 & 8 & & 10 & 15 & & 5 & 38 & \\
\hline & False negative & True negative & 49 & False negative & True negative & 49 & False negative & True negative & 49 \\
\hline
\end{tabular}

\begin{tabular}{|c|c|c|c|c|c|c|c|c|c|}
\hline \multirow[t]{10}{*}{$\begin{array}{l}\text { Bio-prosthetic- } \\
\text { AV }\end{array}$} & CT-pseudo or abscess & & & CT-vegetation & & & CT-dehisc & & \\
\hline & True positive & False positive & & True positive & False positive & & True positive & False positive & \\
\hline & 22 & 4 & & 8 & 1 & & 1 & 3 & \\
\hline & 13 & 11 & & 24 & 17 & & 9 & 37 & \\
\hline & False negative & True negative & 50 & False negative & True negative & 50 & False negative & True negative & 50 \\
\hline & TEE-pseudo or abscess & & & TEE-vegetation & & & TEE-dehisc & & \\
\hline & True positive & False positive & & True positive & False positive & & True positive & False positive & \\
\hline & 18 & 2 & & 15 & 3 & & 2 & 1 & \\
\hline & 7 & 8 & & 9 & 8 & & 4 & 28 & \\
\hline & False negative & True negative & 35 & False negative & True negative & 35 & False negative & True negative & 35 \\
\hline
\end{tabular}

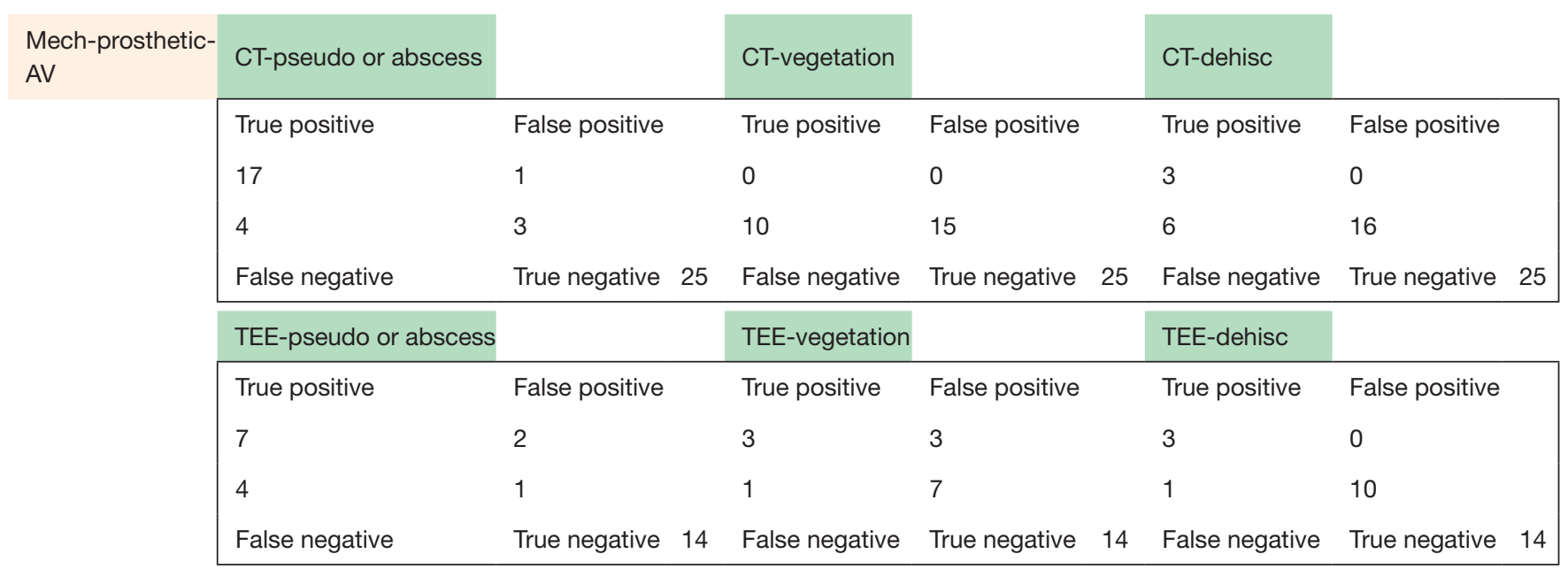

\begin{tabular}{|c|c|c|c|c|c|c|c|c|c|}
\hline \multirow{10}{*}{$\begin{array}{l}\text { Total prosthetic- } \\
\text { MV }\end{array}$} & CT-pseudo or abscess & & & CT-vegetation & & & CT-dehisc & & \\
\hline & True positive & False positive & & True positive & False positive & & True positive & False positive & \\
\hline & 1 & 2 & & 3 & 0 & & 0 & 0 & \\
\hline & 0 & 14 & & 8 & 6 & & 5 & 12 & \\
\hline & False negative & True negative & 17 & False negative & True negative & 17 & False negative & True negative & 17 \\
\hline & TEE-pseudo or abscess & & & TEE-vegetation & & & TEE-dehisc & & \\
\hline & True positive & False positive & & True positive & False positive & & True positive & False positive & \\
\hline & 0 & 0 & & 10 & 2 & & 1 & 0 & \\
\hline & 1 & 12 & & 0 & 1 & & 2 & 10 & \\
\hline & False negative & True negative & 13 & False negative & True negative & 13 & False negative & True negative & 13 \\
\hline
\end{tabular}




\begin{tabular}{|c|c|c|c|c|c|c|c|c|c|}
\hline \multirow{10}{*}{$\begin{array}{l}\text { Bio-prosthetic- } \\
\text { MV }\end{array}$} & CT-pseudo or abscess & & & CT-vegetation & & & CT-dehisc & & \\
\hline & True positive & False positive & & True positive & False positive & & True positive & False positive & \\
\hline & 0 & 2 & & 2 & 0 & & 0 & 0 & \\
\hline & 0 & 10 & & 6 & 4 & & 3 & 9 & \\
\hline & False negative & True negative & 12 & False negative & True negative & 12 & False negative & True negative & 12 \\
\hline & TEE-pseudo or abscess & & & TEE-vegetation & & & TEE-dehisc & & \\
\hline & True positive & False positive & & True positive & False positive & & True positive & False positive & \\
\hline & 0 & 0 & & 7 & 2 & & 1 & 0 & \\
\hline & 0 & 10 & & 0 & 1 & & 1 & 8 & \\
\hline & False negative & True negative & 10 & False negative & True negative & 10 & False negative & True negative & 10 \\
\hline
\end{tabular}

\begin{tabular}{|c|c|c|c|c|c|c|c|c|c|}
\hline \multirow{10}{*}{$\begin{array}{l}\text { Mech- } \\
\text { prosthetic-MV }\end{array}$} & CT-pseudo or abscess & & & CT-vegetation & & & CT-dehisc & & \\
\hline & True positive & False positive & & True positive & False positive & & True positive & False positive & \\
\hline & 1 & 0 & & 1 & 0 & & 0 & 0 & \\
\hline & 0 & 4 & & 4 & 0 & & 2 & 3 & \\
\hline & False negative & True negative & 5 & False negative & True negative & 5 & False negative & True negative & 5 \\
\hline & TEE-pseudo or abscess & & & TEE-vegetation & & & TEE-dehisc & & \\
\hline & True positive & False positive & & True positive & False positive & & True positive & False positive & \\
\hline & 0 & 0 & & 3 & 0 & & 0 & 0 & \\
\hline & 1 & 2 & & 0 & 0 & & 1 & 2 & \\
\hline & False negative & True negative & 3 & False negative & True negative & 3 & False negative & True negative & 3 \\
\hline
\end{tabular}

\begin{tabular}{|c|c|c|c|c|c|c|c|c|c|}
\hline \multirow[t]{5}{*}{ ALL } & \multicolumn{3}{|c|}{ CT + TEE pseudo or abscess } & \multicolumn{3}{|c|}{ CT + TEE -vegetation } & \multicolumn{3}{|c|}{ CT + TEE-dehisc } \\
\hline & True positive & False positive & & True positive & False positive & & True positive & False positive & \\
\hline & 33 & 10 & & 53 & 12 & & 7 & 4 & \\
\hline & 10 & 45 & & 9 & 24 & & 6 & 81 & \\
\hline & False negative & True negative & 98 & False negative & True negative & 98 & False negative & True negative & 98 \\
\hline
\end{tabular}

\begin{tabular}{|c|c|c|c|c|c|c|c|c|c|}
\hline \multirow[t]{5}{*}{ Prosthetic ALL } & \multicolumn{3}{|c|}{ CT + TEE pseudo or abscess } & \multicolumn{3}{|c|}{ CT + TEE -vegetation } & \multicolumn{3}{|c|}{ CT + TEE-dehisc } \\
\hline & True positive & False positive & & True positive & False positive & & True positive & False positive & \\
\hline & 32 & 8 & & 28 & 9 & & 7 & 4 & \\
\hline & 5 & 18 & & 8 & 18 & & 6 & 46 & \\
\hline & False negative & True negative & 63 & False negative & True negative & 63 & False negative & True negative & 63 \\
\hline
\end{tabular}

\begin{tabular}{|c|c|c|c|c|c|c|c|c|c|}
\hline \multirow[t]{5}{*}{ Total prosthetic-AV } & \multicolumn{3}{|c|}{ CT + TEE pseudo or abscess } & \multicolumn{3}{|c|}{ CT + TEE -vegetation } & \multicolumn{3}{|c|}{ CT + TEE-dehisc } \\
\hline & True positive & False positive & & True positive & False positive & & True positive & False positive & \\
\hline & 31 & 6 & & 19 & 7 & & 6 & 4 & \\
\hline & 5 & 7 & & 9 & 14 & & 4 & 35 & \\
\hline & False negative & True negative & 49 & False negative & True negative & 49 & False negative & True negative & 49 \\
\hline
\end{tabular}




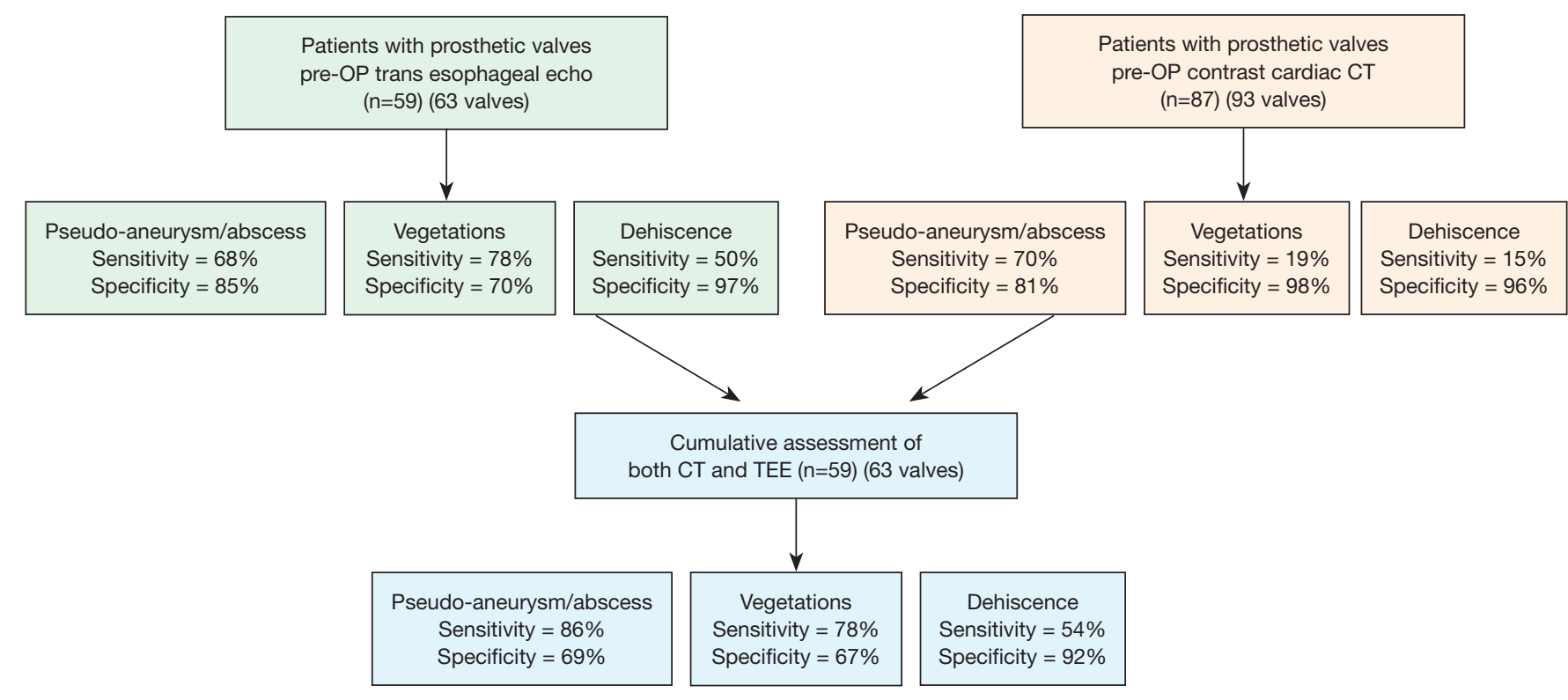

Figure S1 The sensitivity and specificity of detecting pseudoaneurysm/abscess, vegetation, and dehiscence in patients with prosthetic valves with CT, TEE, and CT + TEE combined. CT, computed tomography; TEE, transesophageal echocardiograph.

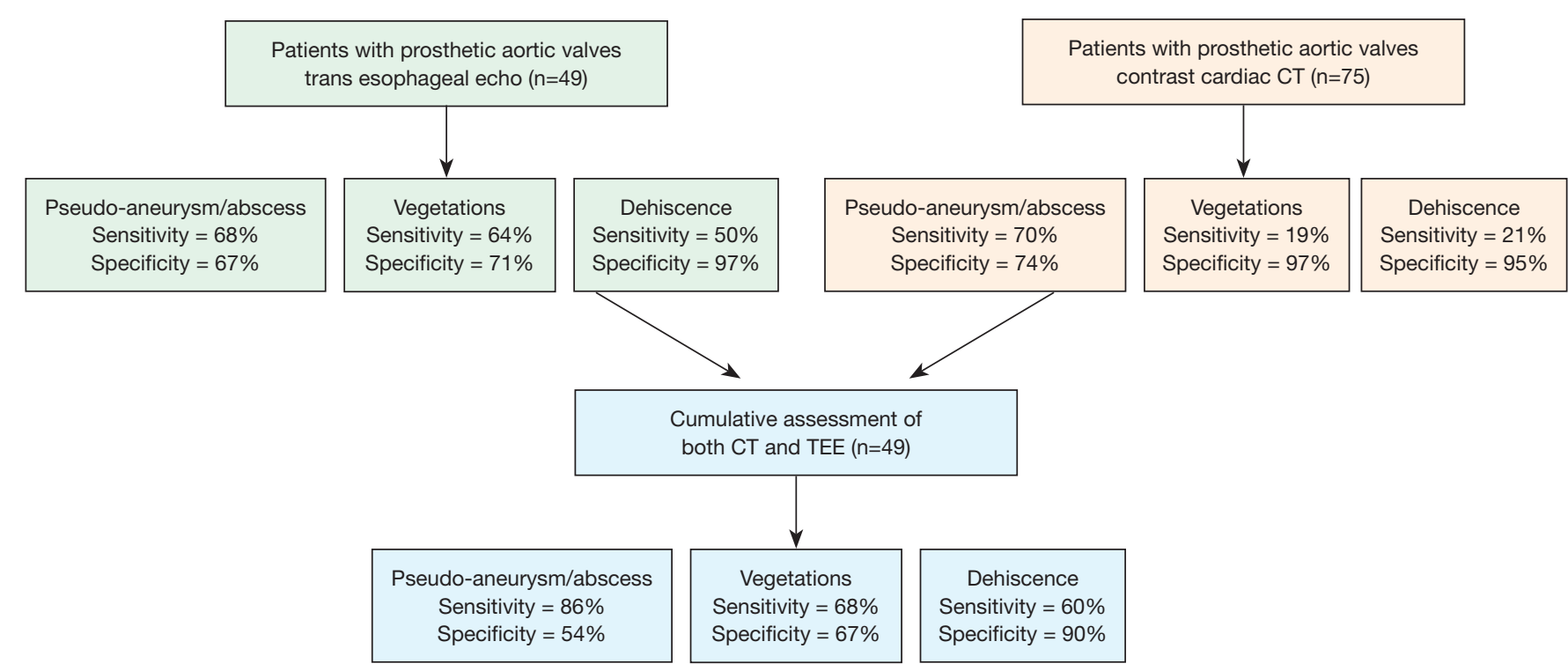

Figure S2 The sensitivity and specificity of detecting pseudoaneurysm/abscess, vegetation, and dehiscence in patients with prosthetic valves in the aortic position with CT, TEE, and CT + TEE combined. CT, computed tomography; TEE, transesophageal echocardiograph.

Table S1 Pathogens identified

\begin{tabular}{lc}
\hline Pathogen & Patients, n [\%] \\
\hline Staphylococcus aureus & $26[21]$ \\
Viridans streptococci & $18[15]$ \\
Coagulase-negative staphylococci & $18[15]$ \\
Enterococcus species & $12[10]$ \\
Pathogen not identified & $12[10]$ \\
Other pathogen & $10[8]$ \\
Nutritionally variant streptococci & $9[7]$ \\
Other gram-positive bacteria & $9[7]$ \\
Fungus & $3[2]$ \\
HACEK microorganism & $2[2]$ \\
Polymicrobial infection & $2[2]$ \\
Propionibacterium acnes & $1[1]$ \\
\hline
\end{tabular}

Data are the number of patients, with percentages in parentheses. HACEK, Haemophilus species, Aggregatibacter actinomycetemcomitans, Cardiobacterium hominis, Eikenella corrodens, and Kingella kingae.
Table S2 CT scanners used in this study and the number of patients scanned on each machine

\begin{tabular}{lc}
\hline CT scanner & Number of patients \\
\hline Phillips Brilliance 64 & 84 \\
Phillips iCT & 16 \\
Siemens Definition 2x64 & 10 \\
Siemens Sensation 64 & 7 \\
Siemens Sensation 16 & 4 \\
Siemens Definition Flash & 1 \\
\hline CT, computed tomography. &
\end{tabular}


Table S3 Evaluation of pseudoaneurysm/abscess, vegetation, and dehiscence by aortic valve position and type

\begin{tabular}{|c|c|c|c|c|c|c|c|c|c|}
\hline \multirow{2}{*}{ Diagnostic performance } & \multicolumn{3}{|c|}{ All prosthetic aortic valves } & \multicolumn{3}{|c|}{ Bioprosthetic aortic valves } & \multicolumn{3}{|c|}{ Mechanical prosthetic aortic valves } \\
\hline & CT $(n=75)$ & TEE $(n=49)$ & $\mathrm{P}$ & CT $(n=50)$ & TEE $(n=35)$ & $P$ & CT $(n=25)$ & TEE $(n=14)$ & $P$ \\
\hline Pseudoaneurysm/abscess (\%) & & & 1 & & & 1 & & & 0.68 \\
\hline Sensitivity & 70 & 68 & & 63 & 72 & & 81 & 64 & \\
\hline Specificity & 74 & 67 & & 73 & 80 & & 75 & 33 & \\
\hline NPV & 45 & 40 & & 46 & 53 & & 43 & 20 & \\
\hline Vegetation (\%) & & & 0.0008 & & & 0.016 & & & 0.04 \\
\hline Sensitivity & 19 & 64 & & 25 & 63 & & 0 & 75 & \\
\hline Specificity & 97 & 71 & & 94 & 73 & & 100 & 70 & \\
\hline Dehiscence (\%) & & & 1 & & & 1 & & & $\mathrm{~N} / \mathrm{A}$ \\
\hline Sensitivity & 21 & 50 & & 10 & 33 & & 33 & 75 & \\
\hline Specificity & 95 & 97 & & 93 & 97 & & 100 & 100 & \\
\hline PPV & 57 & 83 & & 25 & 67 & & 100 & 100 & \\
\hline NPV & 78 & 88 & & 80 & 88 & & 73 & 91 & \\
\hline
\end{tabular}

CT, computed tomography; N/A, not applicable; NPV, negative predictive value; PPV, positive predictive value; TEE, transesophageal echocardiography.

Table S4 Evaluation of pseudoaneurysm/abscess, vegetation, and dehiscence by mitral valve position and type

\begin{tabular}{|c|c|c|c|c|c|c|c|c|c|}
\hline Diagnostic performance & \multicolumn{3}{|c|}{ All prosthetic mitral valves } & \multicolumn{3}{|c|}{ Bioprosthetic mitral valves } & \multicolumn{3}{|c|}{ Mechanical prosthetic mitral valves } \\
\hline Pseudoaneurysm/abscess (\%) & & & 0.47 & & & 0.48 & & & 1 \\
\hline Sensitivity & 100 & 0 & & $\mathrm{~N} / \mathrm{A}$ & $\mathrm{N} / \mathrm{A}$ & & 100 & 0 & \\
\hline Specificity & 88 & 100 & & 83 & 100 & & 100 & 100 & \\
\hline NPV & 100 & 92 & & 100 & 100 & & 100 & 67 & \\
\hline Vegetation (\%) & & & 0.0044 & & & 0.023 & & & 0.24 \\
\hline Sensitivity & 23 & 100 & & 25 & 100 & & 20 & 100 & \\
\hline Specificity & 100 & 33 & & 100 & 33 & & $\mathrm{~N} / \mathrm{A}$ & $N / A$ & \\
\hline Dehiscence (\%) & & & 1 & & & 1 & & & $\mathrm{~N} / \mathrm{A}$ \\
\hline Sensitivity & 0 & 33 & & 0 & 50 & & 0 & 0 & \\
\hline Specificity & 100 & 100 & & 100 & 100 & & 100 & 100 & \\
\hline PPV & $N / A$ & 100 & & $\mathrm{~N} / \mathrm{A}$ & 100 & & $\mathrm{~N} / \mathrm{A}$ & $\mathrm{N} / \mathrm{A}$ & \\
\hline NPV & 71 & 83 & & 75 & 89 & & 60 & 67 & \\
\hline
\end{tabular}

CT, computed tomography; N/A, not applicable; NPV, negative predictive value; PPV, positive predictive value; TEE, transesophageal echocardiography.

Table S5 Incidental findings with CT and TEE

\begin{tabular}{lc}
\hline CT & TEE \\
\hline Aortic dilation/ectasia [29] & Mitral regurgitation [23] \\
Pleural effusions [16] & Aortic regurgitation [21] \\
Left atrial enlargement [16] & Left atrial dilation [9] \\
Lung nodule [14] & Paravalvular regurgitation/leak [6] \\
Left ventricular dilation [13] & Tricuspid regurgitation [5] \\
Splenic infarcts [10] & Aortic stenosis [5] \\
Mediastinal adenopathy [10] & Dilated aorta [5] \\
\hline
\end{tabular}

Number of patients in square bracket. CT, computed tomography; TEE, transesophageal echocardiograph. 